\title{
Geometry Helps to Compare Persistence Diagrams
}

\author{
Michael Kerber $^{*} \quad$ Dmitriy Morozov ${ }^{\dagger} \quad$ Arnur Nigmetov ${ }^{\ddagger}$
}

\begin{abstract}
Exploiting geometric structure to improve the asymptotic complexity of discrete assignment problems is a well-studied subject. In contrast, the practical advantages of using geometry for such problems have not been explored. We implement geometric variants of the Hopcroft-Karp algorithm for bottleneck matching (based on previous work by Efrat el al.) and of the auction algorithm by Bertsekas for Wasserstein distance computation. Both implementations use k-d trees to replace a linear scan with a geometric proximity query. Our interest in this problem stems from the desire to compute distances between persistence diagrams, a problem that comes up frequently in topological data analysis. We show that our geometric matching algorithms lead to a substantial performance gain, both in running time and in memory consumption, over their purely combinatorial counterparts. Moreover, our implementation significantly outperforms the only other implementation available for comparing persistence diagrams.
\end{abstract}

\section{Introduction}

The assignment problem is among the most famous problems in combinatorial optimization. Given a weighted bipartite graph $G$ with $(n+n)$ vertices, it asks for a perfect matching with minimal cost. A common cost function is the minimum of the sum of the $q$-th powers of weights of the matching edges, for some $q \geq 1$. We call the solution in this case the $q$-Wasserstein matching and its cost the $q$-Wasserstein distance. As $q$ tends to infinity, the Wasserstein distance approaches the bottleneck distance, by definition the minimum of the maximum edge weight over all perfect matchings. See [9] for a contemporary discussion of the topic with links to applications.

We consider the geometric version of the assignment problem, where the vertices of $G$ are points in a metric space $(X, d)$, and edge weights are determined by the distance function $d$. The metric structure leads to asymptotically improved algorithms that take advantage of data structures for near-neighbor search. This line of research dates back to Efrat et al. [17] for the bottleneck distance and Vaidya [25] for the 1-Wasserstein case. Rich literature has developed since then, mainly focusing on approximation algorithms for Euclidean metrics in low and high dimensions; see [2] for a recent summary. On the other hand, there has been no rigorous study of whether geometry also helps in practice. Our paper is devoted to this question.

We restrict attention to one scenario that motivates our study of the assignment problem. In the field of topological data analysis, the homological information of a data set is often summarized in a persistence diagram. Such diagrams, themselves point sets in $\mathbb{R}^{2}$, capture connectivity of a data set, and, specifically, how the connectivity changes across various scales [15]. Persistence diagrams are stable: small changes in the data cause only small changes in the diagram [11, 12]. Accordingly, the distances between persistence diagrams have received a lot of attention in applications (e.g.,

\footnotetext{
*Graz University of Technology, Graz, Austria kerber@tugraz .at

${ }^{\dagger}$ Lawrence Berkeley National Laboratory, Berkeley, CA, USA dmitriy@mrzv.org

${ }^{\ddagger}$ Graz University of Technology, Graz, Austria nigmetov@tugraz.at
} 
$[1,19,18])$ : where persistence diagrams serve as topological proxies for the input data, distances between the diagrams serve as proxy measures of the similarity between data sets. These distances, in turn, can be expressed as a Wasserstein or a bottleneck distance between two planar point sets, using $L_{\infty}$ as the metric in the plane (see Section 2 for the precise definition and the reduction).

Our contributions. Our contribution is two-fold. First, we provide an experimental study illuminating the advantages of exploiting geometric structure in assignment problems: we compare mature implementations of bottleneck and Wasserstein distance computations for the geometric and purely combinatorial versions of the problem and demonstrate that exploiting the spatial structure improves running time and space consumption for the matching problem. Second, by focusing on the setup relevant in topological data analysis, we provide the fastest implementation for computing distances between persistence diagrams, significantly improving the implementation in the DiONYSUs library [22]. The latter prototypical implementation is the only publicly available software for the problem. Given the importance of this problem in applications, our implementation is therefore addressing a real need in the community. Our code is publicly available. ${ }^{1}$ This paper contains the following specific contributions:

- For bottleneck matchings, we follow the approach of Efrat et al. [17]: they augment the classical combinatorial algorithm of Hopcroft and Karp [20] with a geometric data structure to speed up the search for vertices close to query points. We do not implement their asymptotically optimal but complicated approach. We instead use a k-d tree data structure [4] to prune the search for matching vertices in remote areas (also proposed by the authors). As expected, this strategy outperforms the combinatorial version that linearly scans all vertices. Several careful design choices are necessary to obtain this improvement; see Section 3.

- For Wasserstein matchings, we implement a geometric variant of the auction algorithm, an approximation algorithm by Bertsekas [5]. We use weighted k-d trees, again with the goal to reduce the search range when looking for the best match of a vertex. A data structure similar to ours appears in [3]. Our implementation outperforms a version of the auction algorithm that does not exploit geometry, which we implement for comparison, both in terms of runtime and space consumption. Both our implementations of the auction algorithm dramatically outperform Dionysus, albeit computing approximations rather than the exact answers as the latter. Dionysus uses a variant of the Hungarian algorithm [24]; see Section 4.

- We extend our auction implementation to the case of points with multiplicities, or masses. While this problem can be trivially reduced to the previous one by replacing a multiple point with a suitable number of simple copies, it is more efficient to handle a point with multiplicity as one entity, splitting it adaptively only when fractions are matched to different points. An extension of the auction algorithm to this case has been decribed by Bertsekas and Castañon [7]. We refer to it as auction with integer masses. Our implementation exploits the geometry of the problem in a similar way as the auction for simple points. Handling masses imposes a certain overhead that slows down the computation if the multiplicities are low. However, our experiments show that the advantage of the auction with integer masses becomes apparent already when the average multiplicity is around 10, and the performance gap between the two variants of the auction increases when the average multiplicity increases; see Section 5.

A conference version of this article appeared in ALENEX 2016 [21]. The major novelty of the present version is the discussion of the auction with integer masses in Section 5. Moreover,

\footnotetext{
${ }^{1}$ https://bitbucket.org/grey_narn/hera
} 
we employed a different variant in the (standard) auction algorithm, which improved the running time of the geometric version by more than a magnitude. Technical explanations and updated experimental evaluation compared to [21] are discussed in Section 4.

\section{$2 \quad$ Background}

Assignment problem. Given a weighted bipartite graph $G=(A \sqcup B, E, w)$, with $|A|=n=|B|$ and a weight function $w: E \rightarrow \mathbb{R}_{+}$, a matching is a subset $M \subseteq E$ such that every vertex of $A$ and of $B$ is incident to at most one edge in $M$. These vertices are called matched. A matching is perfect if every vertex is matched; equivalently, a perfect matching is a matching of cardinality $n$; it can be expressed as a bijection $\eta: A \rightarrow B$.

For a perfect matching $M$, the bottleneck cost is defined as $\max \{w(e) \mid e \in M\}$, the maximal weight of its edges. The $q$-Wasserstein cost is defined as $\left(\sum_{e \in M} w(e)^{q}\right)^{1 / q}$; for $q=1$, this is simply the sum of the edge weights. A perfect matching is optimal if its cost is minimal among all perfect matchings of $G$. In this case, the bottleneck or $q$-Wasserstein cost of $G$ is the cost of an optimal matching. If a graph does not have a perfect matching, its cost is infinite. For $q>1$, the $q$-Wasserstein cost can be reduced to the case $q=1$ with the following simple observation.

Proposition 1. The $q$-Wasserstein cost of $G=(A \sqcup B, E, w)$ equals $q$-th root of the 1 -Wasserstein cost of $G^{\prime}=\left(A \sqcup B, E, w^{q}\right)$, where $w^{q}$ means that all edge weights are raised to the $q$-th power.

We call a graph $G=(A \sqcup B, E, w)$ geometric, if there exists a metric space $(X, d)$ and a map $\phi: A \sqcup B \rightarrow X$ such that for any edge $e=(a, b) \in E, w(e)=d(\phi(a), \phi(b))$. In this case, we generally blur the distinction between vertices and their embedding and just assume for simplicity that $A \sqcup B \subset X$. The motivating example of this work is $X=\mathbb{R}^{2}$ and $d(x, y)=\|x-y\|_{\infty}$.

Persistent homology and diagrams. We are concerned with a particular type of assignment problems in this paper. Specifically, we are interested in distances studied by the theory of persistent homology, distances that measure topological differences between objects. In a nutshell, persistent homology records connectivity of objects - connected components, tunnels, voids, and higherdimensional "holes" - across multiple scales. Persistence diagrams summarize this information as two-dimensional point sets with multiplicities. A point $(x, y)$ with multiplicity $m$ represents $m$ features that all appear for the first time at scale $x$ and disappear at scale $y$. Features appear before they disappear, so the points lie above the diagonal $x=y$. The difference $y-x$ is called the persistence of a feature. In addition to the off-diagonal points, the persistence diagram also contains each diagonal point $(x, x)$, counted with infinite multiplicity. These additional points are needed for stability (discussed below) and make the cardinality of every persistence diagram infinite, even if the number of off-diagonal points is finite.

Given two persistence diagrams $X$ and $Y$, their bottleneck distance is defined as

$$
W_{\infty}(X, Y)=\inf _{\eta: X \rightarrow Y} \sup _{x \in X}\|x-\eta(x)\|_{\infty}
$$

where $\eta$ ranges over all bijections and $\|(x, y)\|_{\infty}=\max \{|x|,|y|\}$ is the usual $L_{\infty}$-norm. Similarly, the $q$-Wasserstein distance ${ }^{2}$ is defined as

$$
W_{q}(X, Y)=\left[\inf _{\eta: X \rightarrow Y} \sum_{x \in X}\|x-\eta(x)\|_{\infty}^{q}\right]^{1 / q} .
$$

\footnotetext{
${ }^{2}$ Named after Leonid Vašersteŭn; see A. M. Vershik [26] for the history of this notion.
} 


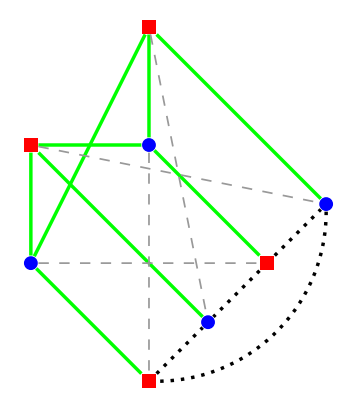

Figure 1: An example of $G$ for two persistence diagrams with 2 off-diagonal points each. Skew edges are dashed gray, edges connecting diagonal points are dotted black.

Why are these distances interesting? Because they are stable $[11,12,14$, Ch. VIII.3]: a small perturbation of the measured phenomenon, for example, a scalar function on a manifold, creates only a small change in the persistence diagram — both distances reflect this. The diagonal of a persistence diagram plays a crucial role in stability. Small perturbations may create new topological features, but their persistence is necessarily small, making it possible to match them to the points on the diagonal. We refer the reader to the cited papers for an extensive discussion.

\section{Persistence distance as a matching problem.}

We assume from now on that persistence diagrams consist of finitely many off-diagonal points with finite multiplicity (and all the diagonal points with infinite multiplicity). In this case, the task of computing $W_{*}(X, Y)$ can be reduced to a bipartite graph matching problem; we follow the notation and argument given in [14, Ch. VIII.4]. Let $X_{0}, Y_{0}$ denote the off-diagonal points of $X$ and $Y$, respectively. If $u=(x, y)$ is an off-diagonal point, we denote its orthogonal projection on the diagonal $((x+y) / 2,(x+y) / 2)$ as $u^{\prime}$, which is the closest point to $u$ on the diagonal. Let $X_{0}^{\prime}$ denote the set of all projections of $X_{0}$, that is $X_{0}^{\prime}=\left\{u^{\prime} \mid u \in X_{0}\right\}$. With $Y_{0}^{\prime}$ defined analogously as $\left\{v^{\prime} \mid v \in Y_{0}\right\}$, we define $U=X_{0} \cup Y_{0}^{\prime}$ and $V=Y_{0} \cup X_{0}^{\prime}$; both have the same number of points. We define the weighted complete bipartite graph, $G=(U \sqcup V, U \times V, c)$, whose weights are given by the function

$$
c(u, v)= \begin{cases}\|u-v\|_{\infty} & \text { if } u \in X_{0} \text { or } v \in Y_{0} \\ 0 & \text { otherwise }\end{cases}
$$

Points from $U$ and $V$ are depicted as squares and circles, respectively, in Figure 1 on the left; all the diagonal points are connected by edges of weight 0 (plotted as dotted lines). The following result is stated as the Reduction lemma in [14, Ch. VIII.4]:

\section{Lemma 2.}

- $W_{\infty}(X, Y)$ equals the bottleneck cost of $G$.

- $W_{q}(X, Y)$ equals the $q$-Wasserstein cost of $G$. This is equal to the q-th root of the 1Wasserstein cost of $G^{q}$, which is the graph $G$ with cost function $c^{q}$, raising all edge costs to the q-th power.

Note that $G$ is almost geometric: distances between vertices are measured using the $L_{\infty}$-metric, except that points on the diagonal can be matched for free to each other if they are not matched with off-diagonal points. Can this almost-geometric structure speed up computation? This question motivates our work. 
It is possible to simplify the above construction. We call an edge $u v \in U \times V$ a skew edge if $u \in X_{0}, v \in X_{0}^{\prime}$ and $v$ is not the projection of $u$, or if $v \in Y_{0}, u \in Y_{0}^{\prime}$ and $u$ is not the projection of $v$ (skew edges are shown with dashed lines in Figure 1).

Lemma 3. For both bottleneck and Wasserstein distance, there exists an optimal matching in $\left(G^{q}, c^{q}\right)$ that does not contain any skew edge.

Proof. Fix an arbitrary matching $M$ and define the matching $M^{\prime}$ as follows: For any $u v \in M \cap$ $X_{0} \times Y_{0}$, add $u v$ and $u^{\prime} v^{\prime}$ to $M^{\prime}$. For any skew edge $a b^{\prime}$ of $M$ with $a$ the off-diagonal point (either in $X_{0}$ or $Y_{0}$ ), add $a a^{\prime}$ to $M^{\prime}$. Also add to $M^{\prime}$ all edges of $M$ of the form $a a^{\prime}$, where $a$ is an off-diagonal point. It is easy to see that $M^{\prime}$ is a perfect matching without skew edges, and its cost is not worse than the cost of $M$ : indeed, the skew edge $a b^{\prime}$ got replaced by $a a^{\prime}$ which is not larger, and the vertices on the diagonal possibly got rearranged, which has no effect on the cost.

Lemma 3 implies that removing all skew pairs does not affect the result of the algorithm, saving roughly a factor of two in the size of the graph. ${ }^{3}$

We prove another equivalent characterization of the optimal cost which will be useful in Section 5: The previous lemma showed that, conceptually, increasing the weight of each skew edge to $\infty$ does not affect the cost of an optimal matching. We show now that even decreasing the weight of a skew edge $a b^{\prime}$ to the weight of $a a^{\prime}$ has no effect on the optimal cost. Formally, let us define $\tilde{G}=(U \sqcup V, U \times V, \tilde{c})$ with a new weight function $\tilde{c}$ as follows:

$$
\tilde{c}(u, v)=\left\{\begin{array}{ll}
\|u-v\|_{\infty} & \text { if } u \in X_{0} \text { and } v \in Y_{0} \\
\left\|u-u^{\prime}\right\|_{\infty} & \text { if } u \in X_{0} \text { and } v \in X_{0}^{\prime} \\
\left\|v-v^{\prime}\right\|_{\infty} & \text { if } u \in Y_{0}^{\prime} \text { and } v \in Y_{0} \\
0 & \text { otherwise }
\end{array} .\right.
$$

Lemma 4. For both bottleneck and Wasserstein distance, there exists an optimal matching in $\tilde{G}$ that does not contain any skew edge.

Proof. The proof of Lemma 3 carries over word by word.

Lemma 5. The weighted graphs $G$ and $\tilde{G}$ have the same bottleneck and Wasserstein cost.

Proof. Let $C$ be the cost for $G$, and $\tilde{C}$ be the cost for $\tilde{G}$ with respect to bottleneck or Wasserstein distance. Since $\tilde{c} \leq c$ edge-wise, $\tilde{C} \leq C$ is immediate. For the opposite direction, fix a matching $\tilde{M}$ that realizes $\tilde{C}$ and has no skew edge (such a matching exists by Lemma 4 ). By the absence of skew edges, the cost $\tilde{M}$ is the same if the cost function $\tilde{c}$ is replaced by $c$. This implies $C \leq \tilde{C}$.

K-d trees. K-d trees [4] are a classical data structure for near-neighbor search in Euclidean spaces. The input point set is split into two halves at the median value of the first coordinates. The process is repeated recursively on the two halves, cycling through the coordinates used for splitting. Each node of the resulting tree corresponds to a bounding box of the points in its subtree. The boxes at any given level are balanced to have roughly the same number of points. Given a query point $q$, one can find its nearest neighbor (or all neighbors within a given radius) by traversing the tree. A subtree can be eliminated from the search if the bounding box of its root node lies farther from the query point than the current candidate for the nearest neighbor (or the query radius). Although the worst case query performance is $O(\sqrt{n})$ in the planar case, k-d trees perform well in practice and are easy to implement. In Section 3 we use the ANN [23] implementation of k-d trees, changing it to support the deletion of points. For Section 4 we implemented our own version of k-d trees to support the search for a nearest neighbor with weights.

\footnotetext{
${ }^{3}$ Dionysus uses the same simplification.
} 


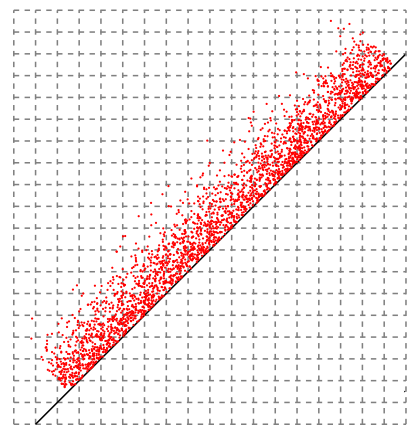

(a) Example of a normal diagram.

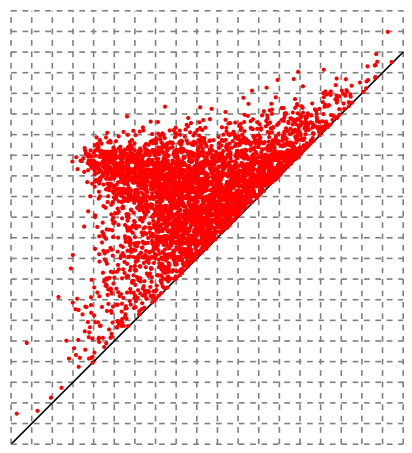

(b) Example of a real diagram.

Figure 2: Examples of persistence diagrams.

Experimental setup. All experiments in the paper were performed on a server running Debian wheezy, with 32 Intel Xeon cores clocked at $2.7 \mathrm{GHz}$, with $264 \mathrm{~GB}$ of RAM. Only one core was used per instance in all our experiments.

We experimentally compare the performance both on artificially generated diagrams as well as on realistic diagrams obtained from point cloud data. For brevity, we restrict the presentation to two classes of instances. In the first class, we generate pairs of diagrams, each consisting of $n$ points. The points are of the form $(a-|b| / 2, a+|b| / 2)$ where $a$ is drawn uniformly in an interval $[0, s]$, and $b$ is chosen from a normal distribution $N(0, s)$, with $s=100$. In this way, the persistence of a point, $|b|$, is normally distributed, so the point set tends to concentrate near the diagonal. This matches the behavior of persistence diagrams of realistic data sets, where points with high persistence are sparse, while the noise present in the data generates the majority of the points, with small persistence. For every set of parameters, we generate 10 independent pairs of diagrams. We refer to this class of experiments as normal instances (Figure 2(a)).

To get a diagram of the second class, we sample a point set $P$ of $n$ points uniformly at random from either a 4-, or a 9-dimensional unit sphere. The 1-dimensional persistence diagram of the Vietoris-Rips filtration of $P$ serves as our input. We use the DIPHA library ${ }^{4}$ for the generation of these instances. Note that persistence diagrams generated in this way have different numbers of points. We refer to this class of experiments as real instances (Figure 2(b)). For each set of parameters (sphere dimension and number of points sampled), we have generated 6 test instances and computed pairwise distances between all $\left(\begin{array}{l}6 \\ 2\end{array}\right)=15$ pairs.

Our plots show the average running times and the standard deviation as error bars. For the real class, the $x$-axis is labelled with the number of points sampled from the sphere, not with the size of the diagram. The size of the persistence diagrams, however, depends practically linearly on the number of sample points, with a constant factor that grows with dimension: the largest instance for dimension 9 is a diagram with 5762 points, while for dimension 4 the largest diagram is of size 1679 .

Our experiments cover many other cases. We have tested various choices of $s$, the scaling parameter in the normal class, and of the sphere dimension in the real class. We have also tried different ways of generating diagrams, for instance, by choosing $n$ points uniformly at random in the square $[0, s] \times[0, s]$, above the diagonal. In all these cases, we encountered the same qualitative difference between the tested algorithms as for the two representative cases discussed in this paper.

\footnotetext{
${ }^{4}$ https://github.com/DIPHA/dipha
} 


\section{Bottleneck matchings}

Our approach follows closely the work of Efrat et al. [17], based on the following simple observation. Let $G[r]$ be the subgraph of $G$ that contains the edges with weight at most $r$. The bottleneck distance of $G$ is the minimal value $r$ such that $G[r]$ contains a perfect matching. Since the bottleneck cost for $G$ must be equal to the weight of one of the edges, we can find it exactly by combining a test for a perfect matching with a binary search on the edge weights.

The algorithm by Hopcroft and Karp. Efrat et al. modify the algorithm by Hopcroft and Karp [20] to find a maximum matching. We briefly summarize the Hopcroft-Karp algorithm; [17] provides an extended review. For a given graph $G[r]$, the algorithm computes a maximum matching, i.e., a matching of maximal cardinality. $G[r]$, with $2 n$ vertices, has a perfect matching if and only if its maximum matching has $n$ edges.

The algorithm maintains an initially empty matching $M$ and looks for an augmenting path, i.e., a path in $G[r]$ that alternates between edges inside and outside of $M$, with the first and the last edge not in $M$. Switching the state of all edges in an augmenting path (inserting or removing them from $M$ ) augments the matching, increasing its size by one.

The algorithm detects several vertex-disjoint augmenting paths at once. It computes a layer subgraph of $G[r]$, from which it reads off the vertex-disjoint augmenting paths. Both the construction of the layer subgraph and the search for augmenting paths are realized through a graph traversal in $G[r]$ in $O(m)$ time, where $m$ is the number of edges. Having identified augmenting paths, the algorithm augments the matching and starts over, repeating the search until all vertices are matched or no augmenting path can be found. As shown in [20], the algorithm terminates after $O(\sqrt{n})$ rounds, yielding a running time of $O(m \sqrt{n})=O\left(n^{2.5}\right)$.

Geometry helps. The crucial observation of Efrat et al. is that for a geometric graph $G[r]$, the layer subgraph does not have to be constructed explicitly. Instead one may use a near-neighbor search data structure, denoted by $\mathcal{D}_{r}(S)$, which stores a point set $S$ and a radius $r$. It must answer queries of the form: given a point $q \in \mathbb{R}^{2}$, return a point $s \in S$ such that $d(q, s) \leq r . \mathcal{D}_{r}(S)$ must support deletions of points in $S$. As the authors show, if $T(|S|)$ is an upper bound for the cost of one operation in $\mathcal{D}_{r}(S)$, the algorithm by Hopcroft and Karp runs in $O\left(n^{1.5} T(n)\right)$ time for a graph with $2 n$ vertices. For the planar case, Efrat et al. show that one can construct such a data structure (for any $L_{p}$-metric) in $O(n \log n)$ preprocessing time, with $T(n)=O(\log n)$ time per operation. Thus, the execution of the Hopcroft-Karp algorithm costs only $O\left(n^{1.5} \log n\right)$.

Naively sorting the edge weights and binary searching for the value of $r$ takes $O\left(n^{2} \log n\right)$ time. But this running time would dominate the improved Hopcroft-Karp algorithm. In order to improve the complexity of the edge search, the authors use an approach, attributed to Chew and Kedem [10], for efficient $k$-th distance selection for a bi-chromatic point set under the $L_{\infty}$-distance; see $[17$, Sec.6.2.2] for details.

With this technique, the computation of a maximum matching dominates the cost of finding the $k$-th largest distance, giving the runtime complexity of $O\left(n^{1.5} \log ^{2} n\right)$ for computing the bottleneck matching. Using further optimizations [17, Sec.5.3], they obtain a running time of $O\left(n^{1.5} \log n\right)$ for geometric graphs in $\mathbb{R}^{2}$ with the $L_{\infty}$-metric.

It is not hard to see that the analysis carries over to the case of persistence diagrams (also mentioned in [14, p.196]). Let $G_{1}=(U \sqcup V, U \times V)$ be the graph defined in Lemma 2 . In the algorithm, $\mathcal{D}_{r}(S)$ is initialized with the points in $V$, which are subsequently removed from it. We additionally maintain a set $S^{\prime}$ of diagonal points contained in $S$. When the algorithm queries a near neighbor of a diagonal point of $U$, we return one of the diagonal points from $S^{\prime}$ in constant time, if $S^{\prime}$ is not empty. The overhead of maintaining $S^{\prime}$ is negligible. We summarize: 


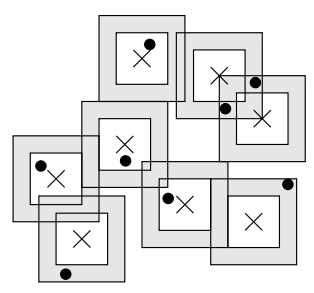

Figure 3: Illustration of the exact computation step: the exact bottleneck distance must be realized by a point in $B$ (circles) in an annulus around $A$ (crosses). The width of the annulus is determined by the approximation quality. In this example, there are 6 candidate pairs.

Theorem 6. The bottleneck distance of two persistence diagrams can be computed in $O\left(n^{1.5} \log n\right)$.

Our approach. Our implementation follows the basic structure of Efrat et al., reducing the construction of layered subgraphs to operations on a near-neighbor data-structure $\mathcal{D}_{r}(S)$. But instead of the rather involved data structure proposed by the authors, we use a simpler alternative: we construct a k-d tree for $S$. When searching for a point at most $r$ away from a query point $q$, we traverse the $\mathrm{k}$ - $\mathrm{d}$ tree, pruning from the search the subtrees whose enclosing box is further away from the query than the current best candidate. When a point is removed from $S$, we mark it as removed in the k-d tree; in particular, we do not rebalance the tree after a removal. We also keep track of how many points remain in each subtree, so that we can prune empty subtrees from the subsequent searches. The running time per search query can be bounded by $O(\sqrt{n})$ per query, with $n$ the number of points originally stored in the search tree. We remark that using range trees [13], the worst-case complexity could be further reduced to $O(\log n)$.

Initial tests showed that the naive approach of precomputing and sorting all distances for the binary search dominates the running time in practice. Instead of implementing the asymptotically fast but complicated approach of Efrat et al., we compute a $\delta$-approximation of the bottleneck distance, which we can then post-process to compute the exact answer. Let $d_{\text {max }}$ denote the maximal $L_{\infty}$-distance between a point in $U$ and a point in $V$ in $G_{1}$. First, we compute, in linear time, a 3-approximation of $d_{\max }$ as follows. We pick an arbitrary point in $U$, find its farthest point $v_{0} \in V$, and find a point $u_{0} \in U$ farthest from $v_{0}$. Then, $\left\|u_{0}-v_{0}\right\|_{\infty} \leq d_{\max } \leq 3\left\|u_{0}-v_{0}\right\|_{\infty}$ (from the triangle inequality). Setting $t=3\left\|u_{0}-v_{0}\right\|_{\infty}$, the exact bottleneck distance $o$ must be in $[0, t]$ and we perform a binary search on $[0, t]$ until we find an interval $(a, b]$ that satisfies $(b-a)<\delta \cdot a$. We return $b$ as the approximation. It is easy to see that $b \in[o,(1+\delta) o)$.

At each iteration of the binary search, we reuse the maximum matching constructed before (if the true distance is below the midpoint of the current interval $(a, b]$, we remove edges whose weight is greater than $(a+b) / 2$, otherwise the whole matching can be kept).

To get the exact answer, we find pairs in $U \times V$ whose distance is in the approximation interval, $(a, b]$. For such a pair $(u, v), v$ lies in an $L_{\infty}$-annulus around $u$ with inner radius $a$ and outer radius $b$. So we find for every $u \in U$ the points of $V$ in the corresponding annulus and take the union of all such pairs as the candidate set. In the example in Figure 3, points in $U$ are drawn as crosses, points in $V$ as circles, and there are 6 candidate pairs.

We compute the candidate pairs with similar techniques as used for range trees [13]. Specifically, we identify all pairs $(u, v)$ whose $x$-coordinate difference lies in $(a, b]$. We can compute the set $C_{x}$ of such pairs in $O\left(n \log n+\left|C_{x}\right|\right)$ time by sorting $U$ and $V$ by $x$-coordinates. For each pair $(u, v)$ in $C_{x}$, we check in constant time whether $\|u-v\|_{\infty} \in(a, b]$ and remove the pair otherwise. We then repeat the same procedure using the $y$-coordinates. To compute the exact bottleneck distance, we perform binary search on the vector of candidate distances. 

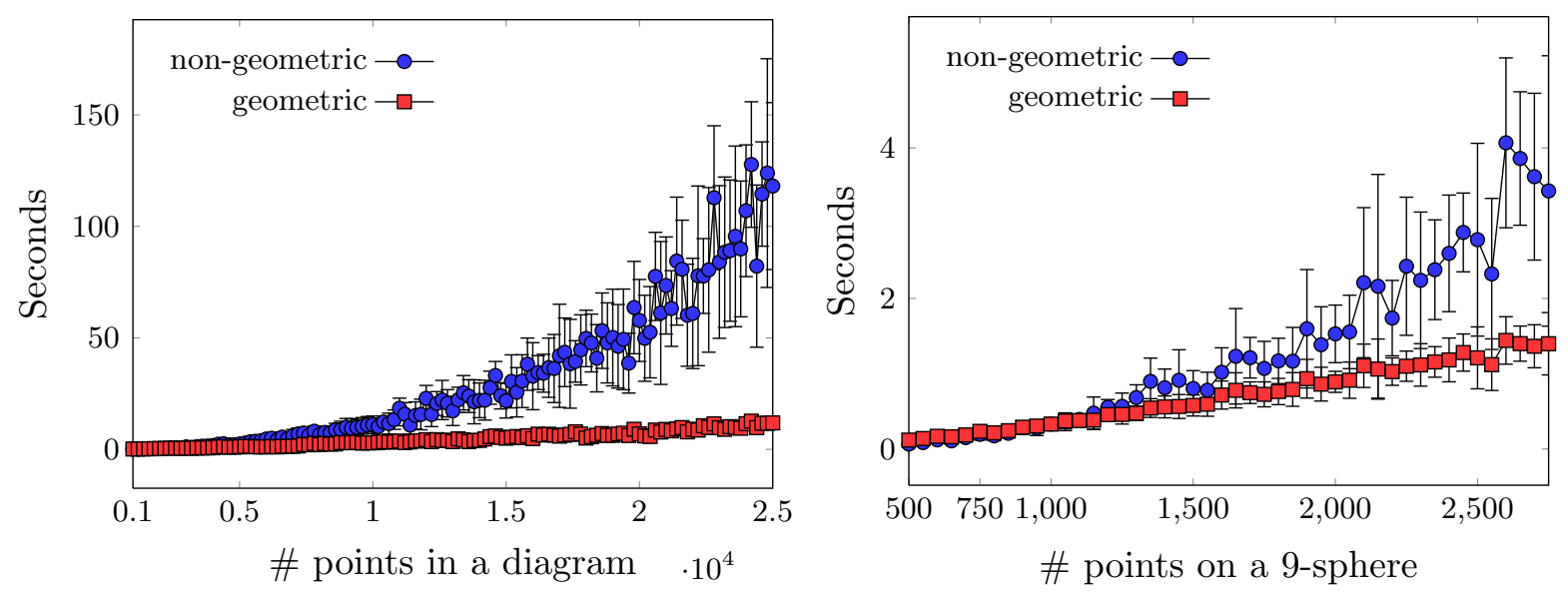

Figure 4: Running times of the bottleneck distance computation on normal data (left) and real data (right) for varying number of points.

Let $c$ denote the number of candidate pairs. The complexity of our procedure is not outputsensitive in $c$ because $\left|C_{x}\right|+\left|C_{y}\right|$ can be larger than $c$ - so too many pairs might be considered. Nevertheless, we expect that when using a sufficiently good initial approximation, both $\left|C_{x}\right|+\left|C_{y}\right|$ and $c$ are small, so our method will be fast in practice.

Experiments. We compare the geometric and non-geometric bottleneck matching algorithms. We set $\delta=0.01$ and compute the approximate bottleneck distance to the relative precision of $\delta$, using k-d trees for the geometric version and constructing the layered graph combinatorially in the non-geometric version. Figure 4 shows the results for normal and real instances. We observe that the geometric version scales significantly better, and runs faster by a factor of roughly 10 for the largest displayed normal instance with 25000 points per diagram. We remark that the memory consumption of the geometric and non-geometric versions both scale linearly, and the geometric version is larger by a factor of roughly 4 throughout. For 25000 points, about $60 \mathrm{MB}$ of memory is required.

We used linear regression to fit curves of the form $c n^{\alpha}$ to the plots of Figure 4 (left). For the non-geometric version, the best fit appeared for $\alpha=2.3$, roughly matching the asymptotic bound of Hopcroft-Karp. For the geometric version, we get the best fit for $\alpha=1.4$; this shows that despite the pessimistic worst-case complexity, the algorithm tends to follow the improved geometric bound on practical instances.

The above experiment does not include the post-processing step of computing the exact bottleneck distance. We test the geometric version above that yields a $1 \%$ approximation against the variant that also computes the exact distance from the initial approximation, as explained earlier in this section. Our experiments show that the running time of the post-processing step is about half of the time needed to get the approximation. Although there is some variance in the ratio, it appears that the post-processing does not worsen the performance by more than a factor of two.

Figure 5 compares our exact (geometric) bottleneck algorithm with DiONYSUs, the only publicly available implementation for computing bottleneck distance between persistence diagrams. DiONYSUS simply sorts the edge distances in increasing order and performs a binary search, building the graphs $G[r]$ and calling the Edmonds matching algorithm [16] from the Boost library to check for a perfect matching in $G[r]$. Already for diagrams of 2800 points, our speed-up exceeds a factor of 400. 

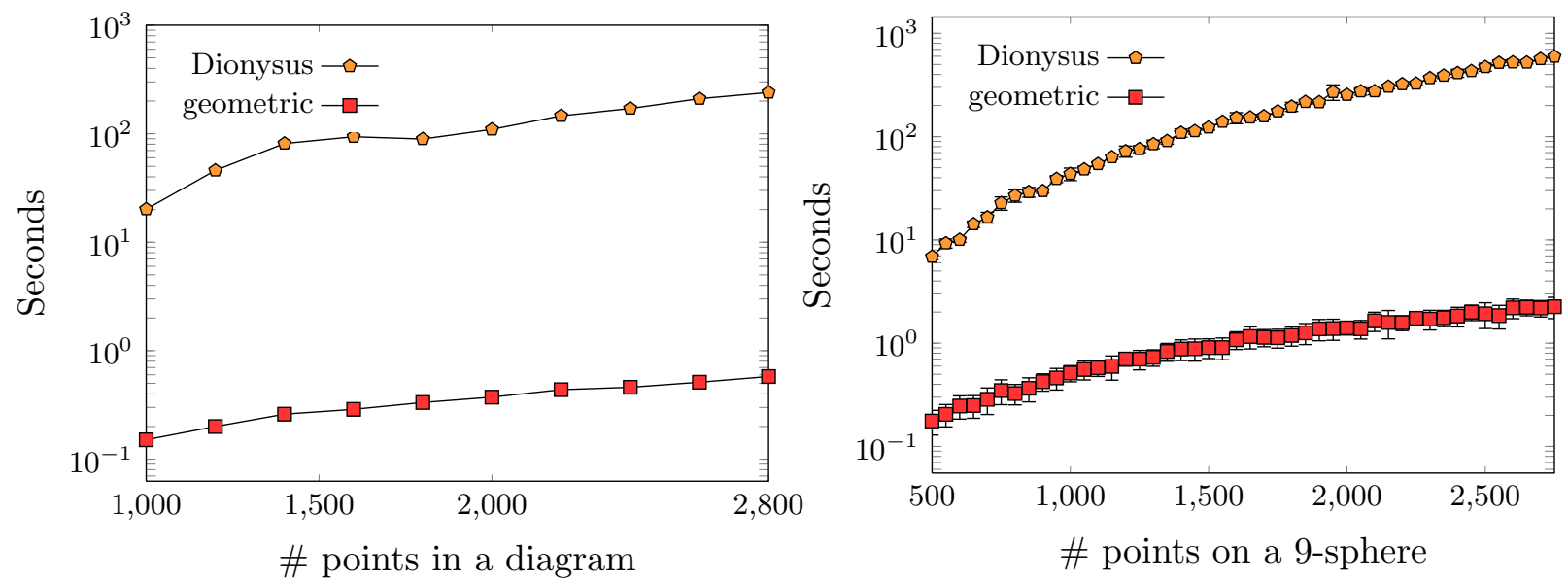

Figure 5: Comparison of our exact geometric bottleneck algorithm with DiONYSUs for normal (left) and real (right) input.

\section{$4 \quad$ Wasserstein matchings}

We now fix $q \geq 1$ and describe an algorithm for computing the $q$-Wasserstein cost of a weighted graph $(U \sqcup V, E, w)$. Recall from Proposition 1 that we can restrict to the 1-Wasserstein case by switching to the cost function $w^{q}$. Moreover, we assume that $U=\left\{u_{1}, \ldots, u_{n}\right\}$ and $V=\left\{v_{1}, \ldots, v_{n}\right\}$ are finite sets, and we identify the elements with their indices.

Auction algorithm. The auction algorithm of Bertsekas [5] is an asymmetric approach to find a perfect matching in a weighted graph that maximizes the sum of its edge weights. One half of the bipartite graph is treated as "bidders", the second half as "objects." Initially, each object $j$ is assigned zero price, $p_{j}=0$, and each bidder $i$ extracts a certain benefit, $b_{i j}$, from object $j$. Since we are interested in the minimum cost matching, we use the negation of the edge weight as the bidder-object benefits, that is, $b_{i j}=-w^{q}(i, j)$. If the edge $(i, j)$ is not in the graph, $b_{i j}=-\infty$. The auction algorithm maintains a (partial) matching $M$, which is empty initially. When $M$ becomes perfect, the algorithm stops. During the execution of the algorithm, matched bidders in $M$ are called assigned (to an object), and unmatched bidders are unassigned.

The auction proceeds iteratively. In each iteration, one unassigned bidder $i$ chooses an object $j$ with the maximum value, defined as the benefit minus the current price of the object, $v_{i j}=\left(b_{i j}-p_{j}\right)$. Object $j$ is assigned to the bidder; if it was assigned before, the previous owner becomes unassigned. Let $\Delta p_{i j}$ denote the difference of $v_{i j}$ and the value of the second best object for bidder $i ; \Delta p_{i j}$ can be zero. The price of object $j$ increases by $\Delta p_{i j}+\varepsilon$, where $\varepsilon$ is a small constant needed to avoid infinite loops in cases where two bidders extract the same value from two objects. Without $\varepsilon$, the two could keep stealing the same object from each other without increasing its price.

Our variant of the algorithm is called Gauss-Seidel auction: an iteration consists of only one bid, which is always satisfied. An alternative, called the Jacobi auction, proceeds by letting each unassigned bidder place a bid in every iteration. If several bidders want the same object, it is assigned to the bidder who offers the highest price increment, $\Delta p_{i j}+\varepsilon$. The Jacobi auction, which was used in the ALENEX version of this paper [21], has a drawback if many objects provide the same value to many bidders. In that case, it may happen that all of these bidders bid for the same object in one iteration, and all but one of them remain unassigned. Since a Jacobi iteration is more expensive than a Gauss-Seidel iteration, this may result in worse performance. Indeed, 
our experiments show that switching to Gauss-Seidel auction improves the runtime by an order of magnitude.

How do we choose $\varepsilon$ ? Small values give a better approximation of the exact answer; on the other hand, the algorithm converges faster for large values of $\varepsilon$. Bertsekas suggests $\varepsilon$-scaling to overcome this problem: running several rounds of the auction algorithm with decreasing values of $\varepsilon$, using prices from the previous round, but an empty matching, as an initialization for the next round. Following the recommendation of Bertsekas and Castañon [8], we initialize $\varepsilon$ with the maximum edge cost divided by 4 and divide $\varepsilon$ by 5 when starting a new round.

Iterating this procedure long enough would eventually yield the exact Wasserstein distance [5]; however, the number of rounds of $\varepsilon$-scaling would in general be too high for many practical problems. Instead, we use a termination condition that guarantees a relative approximation of the exact value. We fix some approximation parameter $\delta \in(0,1)$. After finishing a round of the auction algorithm for $q$-Wasserstein matching for some value $\varepsilon>0$, let $d:=d_{\varepsilon}$ be the $q$-th root of the cost of the obtained matching. We stop if $d$ satisfies

$$
d^{q} \leq(1+\delta)^{q}\left(d^{q}-n \varepsilon\right)
$$

and return $d$ as the result of the algorithm. We summarize the auction in Algorithm 1.

Lemma 7. The return value $d$ of the algorithm satisfies

$$
d \in[o,(1+\delta) o)
$$

where o denotes the exact $q$-Wasserstein distance.

Proof. Because we raise all edge costs to the $q$-th power, the matching minimizing the sum of the edge costs has a cost of $o^{q}$. Let $d^{q}$ be the cost of the matching computed by the auction algorithm, after the last round of $\varepsilon$-scaling, for a fixed $\varepsilon$. By the properties of the auction algorithm ([6], Proposition 1), it holds (after every round) that

$$
o^{q} \leq d^{q} \leq o^{q}+n \varepsilon .
$$

Taking the $q$-th root yields the first inequality immediately. For the second inequality, note that

$$
(1+\delta)^{q} o^{q} \geq(1+\delta)^{q}\left(d^{q}-n \varepsilon\right) \geq d^{q},
$$

where the last inequality follows from the termination condition of the algorithm. Taking the $q$-th root on both sides yields the result.

Bidding. The computational crux of the algorithm is for a bidder to determine the object of maximum value and the price increase. The brute-force approach is for each bidder to do an exhaustive search over all objects. Doing so requires linear running time per iteration. But let us consider what the search actually entails. Bidder $i$ must find the two objects with highest and second-highest $v_{i j}$ values. Recall $v_{i j}=b_{i j}-p_{j}=-w^{q}(i, j)-p_{j}$, and maximizing this quantity for a fixed $i$ is equivalent to minimizing $w^{q}(i, j)+p_{j}$.

The first way to quickly find these objects uses lazy heaps. Each bidder keeps all the objects in a heap, ordered by their value. We also maintain a list of all the price changes (for any object), as well as a record for each bidder of the last time its heap was updated. Before making a choice, a bidder updates the values of all the objects in its heap that changed prices since the last time the heap was updated. The bidder then selects the two objects with the maximum value. We note that this approach uses quadratic space, since each bidder keeps a record of each object. 


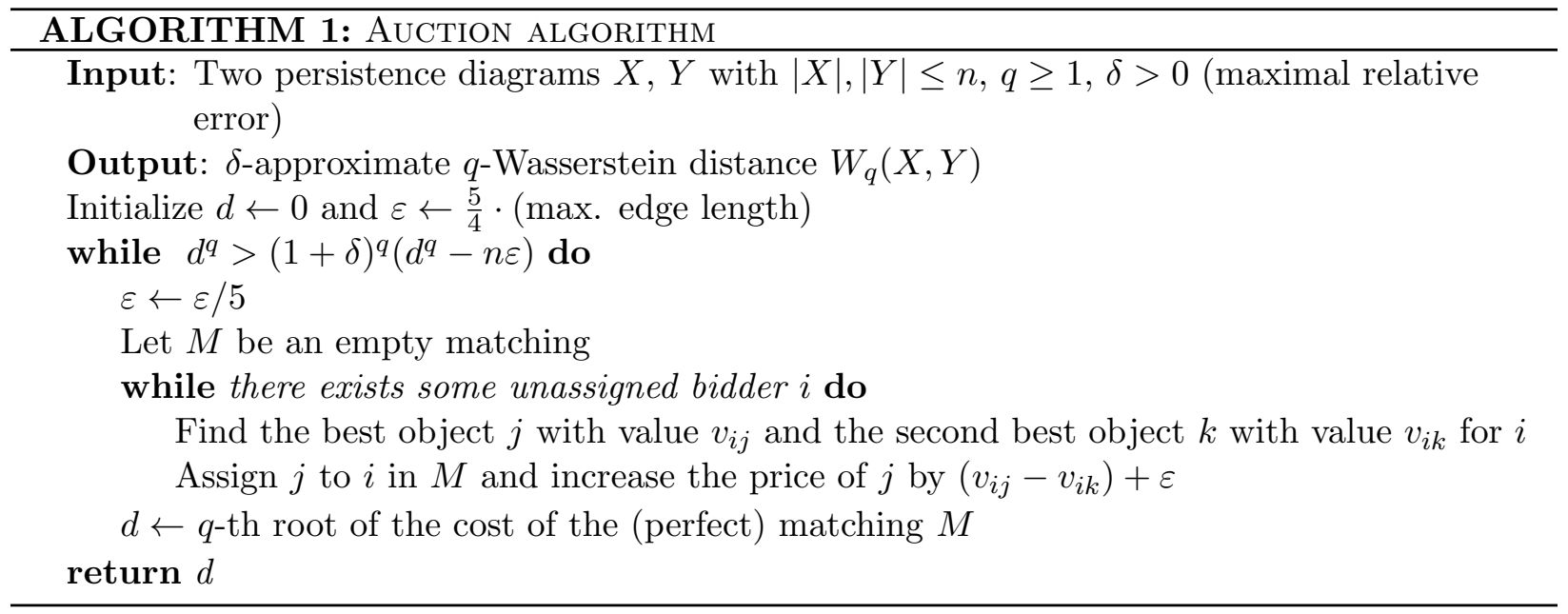

The second way to accelerate the search for the best object uses geometry and requires only linear space. Initially, when all the prices are zero, we can find the two best objects by performing the proximity search in a k-d tree. But we need to augment the $\mathrm{k}$ - $\mathrm{d}$ tree to take increasing prices into account. We do so by storing the price of each point as its weight in the $\mathrm{k}$-d tree. At each internal node of the tree we record the minimum weight of any node in its subtree. When searching, we prune subtrees if the $q$-th power of the distance from the query point to the box containing all of the subtree's points, plus the minimum weight in the subtree, exceeds the current second best candidate.

Once a bidder selects the best object, it increases its price. We adjust the subtree weights in the k-d tree by increasing the chosen object's weight and updating the weights on the path to the root. If the minimum weight does not change at some node on the path, we interrupt the traversal.

The case of persistence diagrams requires special care. We can distinguish between diagonal and off-diagonal bidders and objects. Diagonal bidders should bid for only one off-diagonal object, according to Lemma 3. Since the distance between diagonal points is 0 , the value of a diagonal object $j$ for a digonal bidder $i$ is just the opposite of its price, $v_{i, j}=-p_{j}$, and we keep all diagonal objects in a heap ordered by the price. When a diagonal bidder needs to find the best two objects, it selects the top two elements of the heap and compares them with the only off-diagonal object to which it can be assigned.

On the other hand, off-diagonal bidders can bid for every off-diagonal object and only for one diagonal object (its projection). We use one global k-d tree to get the best two off-diagonal objects and then compare their values for the bidder with the value of bidder's projection, so only off-diagonal objects are stored in the k-d tree.

Experiments. Figure 7 illustrates the running times of the auction algorithm on the normal data, using lazy heaps and k-d trees. In both cases, we compute a relative 0.01-approximation. The advantage of using geometry is evident: the algorithm is faster by roughly a factor of 4 for diagrams with 1000 points, and the factor becomes close to 20 for diagrams with 10000 points. We used linear regression to empirically estimate the complexity, and the geometric algorithm runs in $O\left(n^{1.6}\right)$, while for the non-geometric algorithm the estimated complexity is super-quadratic, $O\left(n^{2.3}\right)$. The non-geometric version only shows competitive running times because of the described optimization with lazy heaps. This results in a severe increase in memory consumption, as displayed in Figure 6.

Again, we compare our geometric approach with Dionysus, which uses John Weaver's imple- 


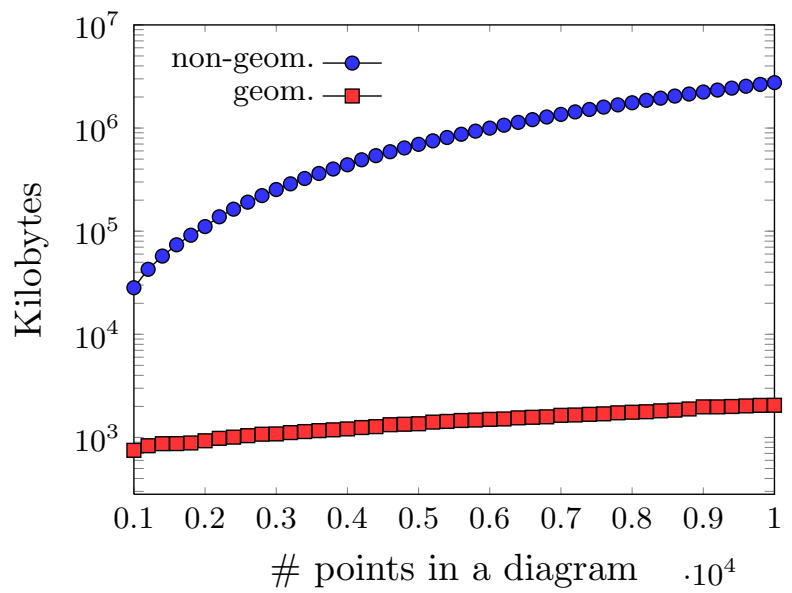

Figure 6: Comparison of memory consumption of geometric and non-geometric versions of auction algorithm on normal instances.
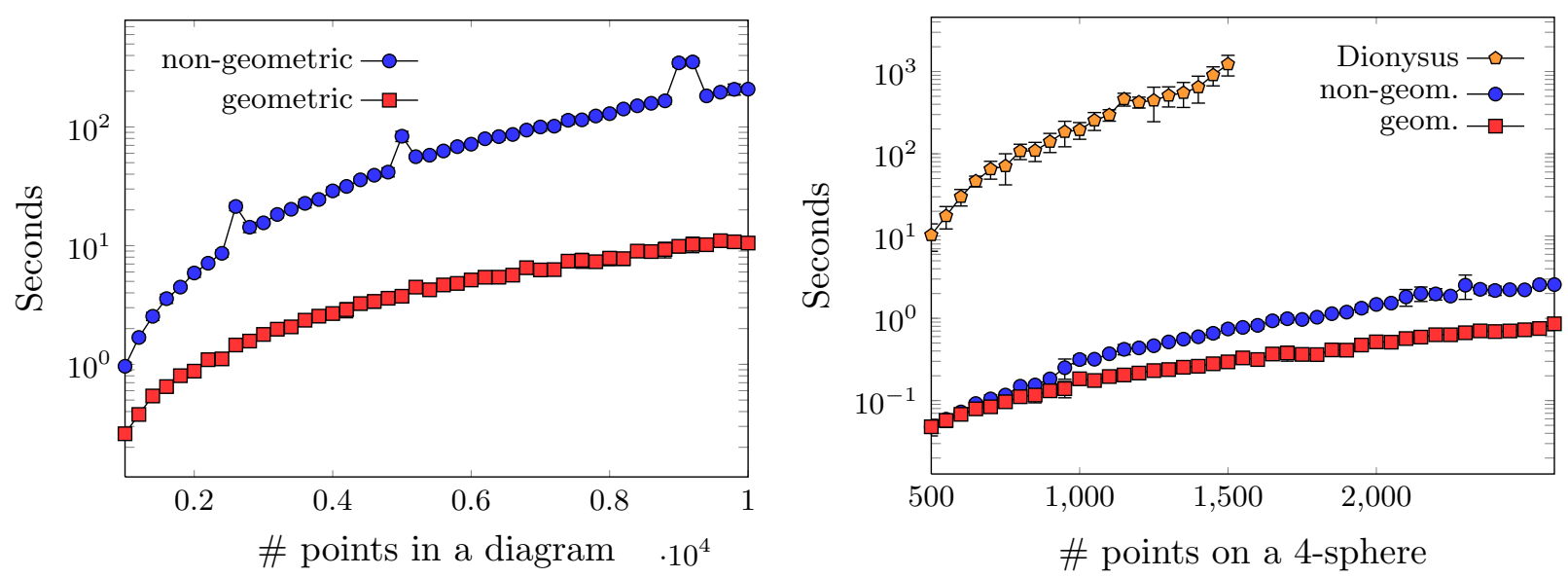

Figure 7: Comparison of non-geometric and geometric variants of the auction algorithm on normal (left) and real (right) input, also with Dionysus on the real input.

mentation $^{5}$ of the Hungarian algorithm [24]. Figure 7 (right) shows the results for real instances. The speed-up of our approach increases from a factor of 50 for small instances to a factor of about 400 for larger instances. For the normal data sets, the speed-up already exceeds a factor of 1000 for diagrams of 1000 points; we therefore omit a plot.

We emphasize that our test is slightly unfair, as it compares the exact algorithm from DionYsus with the 0.01-approximation provided by our implementation. While such an approximation suffices for many applications in topological data analysis, the question remains how much overhead would be caused by an exact version of the auction algorithm. A naive approach to get the exact result is to rescale the input to integer coordinates and to choose $\varepsilon$ such that the approximation error is smaller than 1. We plan to investigate different possibilities to compute the exact distance more efficiently.

\footnotetext{
${ }^{5}$ http://saebyn.info/2007/05/22/munkres-code-v2/
} 


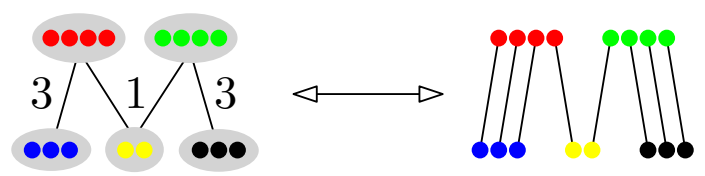

Figure 8: Correspondence between assignments and matchings. On the left-hand side there are two multi-bidders, each of mass 4 , and 4 slices with masses $3,3,1,1$. A corresponding perfect matching is on the right-hand side.

\section{$5 \quad$ Wasserstein matchings for repeated points}

For a weighted, complete, bipartite graph $G=(U \sqcup V, U \times V, w)$, we call two vertices $u_{1}, u_{2} \in$ $U$ identical if for all $v \in V, w\left(u_{1}, v\right)=w\left(u_{2}, v\right)$. A pair of identical vertices in $V$ is defined symmetrically. If $G$ is a geometric graph, two points with coinciding locations are identical. In the context of persistence diagrams, this situation is common in applications, where the range of possible scales on which features appear and disappear is often discretized. The discretization places all points of the persistence diagram on a finite grid. For a fixed discretization of a fixed range, more and more identical points appear as the data size grows. This raises the question whether diagrams with many identical points can be handled more efficiently.

Auction with integer masses. We use a variant of the auction algorithm [7], which we explain next. The input consists of two sets $U$ and $V$ of multi-points, each given by its coordinates and integer multiplicity $m \geq 1$; a multi-point represents $m$ identical points at the given location. For brevity, we refer to the multiplicity as mass. The total mass of both sets is the same. In analogy to the auction algorithm from Section 4, we refer to the elements of the respective sets as multibidders and multi-objects. The elements of a multi-object are not, in general, assigned to the same multi-bidder; their prices can also differ. However, if two elements of a multi-object are assigned to one multi-bidder, the algorithm guarantees that their prices are equal. The algorithm decomposes a multi-object into slices, where each slice represents a fraction of the multi-object that is currently not distinguished by the algorithm. Formally, a slice is a four-tuple $\left(j, m_{i, j}, p_{i, j}, i\right)$ identifying the multi-object $j$ it belongs to, the mass of the slice $m_{i, j}$, its price $p_{i, j}$, and the multi-bidder $i$ that it is currently assigned. The decomposition of multi-objects into slices defines an assignment, which can be interpreted as a matching $M$ in the original graph (see Fig. 8): A slice $\left(j, m_{i, j}, p_{i, j}, i\right)$ corresponds to $m_{i, j}$ edges in $M$ from $m_{i, j}$ elements of the multi-bidder $i$ to $m_{i, j}$ elements of the multiobject $j$ (hereby interpreting multi-bidders and multi-objects as sets of identical bidders/objects). Unassigned slices correspond to unmatched vertices. We call an assignment perfect if the induced matching is perfect, and the cost of the assignment is the cost of the corresponding matching.

The auction with integer masses is a procedure converging to an assignment with minimal cost. It uses the same high-level structure as the auction described in Section 4, which we will refer to as the standard auction. It employs $\varepsilon$-scaling with the same choices of parameters. One round of $\varepsilon$-scaling maintains an assignment and runs until the assignment is perfect, that is, all multibidders are fully assigned to multi-objects. Every round proceeds in iterations. In each iteration, one multi-bidder with unassigned mass is selected at random. It acquires enough slices (possibly taking them away from other multi-bidders) to assign all its missing mass and increases the prices of these slices.

Specifically, an iteration proceeds as follows. We fix a multi-bidder with some unassigned mass $u \geq 1$, and let $s_{1}, \ldots, s_{t}$ be the slices assigned to it. Conceptually, the algorithm takes all possible slices except for $s_{1}, \ldots, s_{t}$ and sorts them by their value to the multi-bidder in decreasing order. We denote the sorted slices by $s_{t+1}, \ldots, s_{N}$; let $v_{i}$ denote the value of $s_{i}$ to the multi-bidder. 
The multi-bidder takes the first $k$ slices $s_{t+1}, \ldots, s_{t+k}$ such that their total mass $m$ is at least $u$. If $m>u$, we split the "leftover" slice from $s_{t+k}$ whose mass is $m-u$ and whose price and owner remain unchanged; we denote this newly created slice as $\tilde{s}_{t+k}$. Now, the total mass of the slices $s_{t+1}, \ldots, s_{t+k}$ is exactly $u$, and we assign them to the multi-bidder.

Next, we increase the prices of all $t+k$ slices assigned to the multi-bidder. Let $s_{l}$ with $l \geq t+k$ be a slice determined as follows: if the slices $s_{1}, \ldots, s_{t+k}$ belong to at least two different multiobjects, $s_{l}$ is the slice containing the $(m+1)$-st unit of mass, that is, $s_{l}$ is set to $s_{t+k+1}$ if we did not split the leftover slice, and to $\tilde{s}_{t+k}$ otherwise. If all the $t+k$ slices are of a single multi-object, then $s_{l}$ is defined to be the first slice among $s_{t+k+1}, \ldots, s_{N}$ that belongs to a different multi-object. Let $v_{l}$ be the value of $s_{l}$ to the multi-bidder. We increase the prices of the slices $\left\{s_{i}\right\}_{1 \leq i \leq t+k}$ by $v_{i}-v_{l}+\varepsilon$ to make them as valuable to the multi-bidder as the slice $s_{l}$, up to $\varepsilon$.

The original paper that presents this approach [7] describes the Jacobi version of the algorithm, i.e., all bidders with unassigned mass submit bids in one iteration, and the mass goes to the bidder who offered the highest bid. The above description is the Gauss-Seidel variant of the same algorithm, and it is straightforward to verify that the same proof of correctness works for it, too. From the discussion in [7], it follows that one can use the same formula as for the standard auction to estimate the relative error of the matching obtained after each round of $\varepsilon$-scaling. Therefore, we can use the same termination condition as in (3) and the proof of Lemma 7 carries over. We refer to [7] for further details.

Diagonal points. Let $X_{0}$ and $Y_{0}$ be the of the off-diagonal mult-points of two persistence diagrams. Recall that for the computation of the $q$-Wasserstein distance, we introduce the projection sets $Y_{0}^{\prime}$ and $X_{0}^{\prime}$ (also as a set of multi-points with masses inherited from their pre-images) and set $X:=X_{0} \cup Y_{0}^{\prime}$ and $Y:=Y_{0} \cup X_{0}^{\prime}$, which are sets of multi-points with equal total mass. We can run the auction with integer masses, using the cost function $c^{q}$, with $c$ as in (1), and return the $q$-th root of the obtained cost as our result. However, we get a major improvement from using the modified cost function $\tilde{c}$, defined in (2). The modified function decreases the costs of all skew edges; accordingly, $\tilde{c}^{q}$ treats all points in $X_{0}^{\prime}$ as identical and all points in $Y_{0}^{\prime}$ as identical.

In terms of the auction with integer masses, this means that we only need one additional multibidder (with large mass) to represent all projections of multi-objects to the diagonal, and vice versa. Specifically, writing $X_{0}=\left\{x_{1}, \ldots, x_{k}\right\}$ for the off-diagonal multi-bidders, let $m_{X}$ denote their total mass. Let $Y_{0}=\left\{y_{1}, \ldots, y_{\ell}\right\}$ denote the off-diagonal multi-objects with total mass $m_{Y}$. We introduce one additional multi-bidder $Y_{0}^{\prime}:=\left\{x_{k+1}\right\}$ (representing all projections of multi-objects), with mass $m_{Y}$, and one additional multi-object $X_{0}^{\prime}:=\left\{y_{\ell+1}\right\}$ with mass $m_{X}$. The bidder-object benefits are set up according to (2) (recall that $x_{i}^{\prime}$ denotes the projection of $x_{i}$ onto the diagonal):

$$
b_{i, j}= \begin{cases}-\left\|x_{i}-y_{j}\right\|_{\infty}^{q}, & i \leq k \text { and } j \leq \ell \\ -\left\|x_{i}-x_{i}^{\prime}\right\|_{\infty}^{q}, & i \leq k \text { and } j=\ell+1 \\ -\left\|y_{j}-y_{j}^{\prime}\right\|_{\infty}^{q}, & i=k+1 \text { and } j \leq \ell \\ 0, & i=k+1 \text { and } j=\ell+1\end{cases}
$$

Implementation. We implemented a geometric version of the auction with integer masses, where the best slices of the off-diagonal multi-objects are determined using one global k-d tree, similar to Section 4. Here, each leaf of the k-d tree represents a multi-object, and its weight corresponds to the price of its cheapest slice. For a fixed off-diagonal multi-bidder, we can compute an upper bound on the value of all multi-objects stored in a subtree of the $\mathrm{k}$ - $\mathrm{d}$ tree. During a search, we maintain a candidate set of slices whose total mass exceeds the unassigned mass of the multi-bidder, and we can prune a subtree if that upper bound is below the value of the worst candidate. The 
weights in the $\mathrm{k}-\mathrm{d}$ tree are updated as in Section 4. The additional information required to compute the price increases are gathered by similar techniques; we omit the details. We did not implement a non-geometric version using lazy heaps because it would suffer from the same quadratic space complexity as in the standard auction.

Again we need to deal with the diagonal multi-object and multi-bidder separately. We maintain a heap with the slices of the diagonal multi-object sorted by the price and a heap with the slices of all multi-objects (including the diagonal one) sorted by their value for the diagonal bidder. The diagonal bidder finds the best slices by simply traversing the latter heap. An off-diagonal bidder first uses the k-d tree to find the best slices of off-diagonal objects. Then it starts traversing the heap with slices of the diagonal object, replacing the off-diagonal slices with the diagonal ones as long as the diagonal slices offer better values. When the value of the next diagonal slice in the heap is below the minimal value of the currently accumulated slices, we stop traversing the heap with diagonal slices. When slice prices are increased, we immediately update the heaps.

Experiments. As input, we turn the aforementioned instances of normal type into diagrams with integer masses. For each point of the original diagram, we assign mass $m$, drawn uniformly from the range $[\lceil k / 2\rceil,\lfloor 3 k / 2\rfloor]$, so that the average mass of a point is $k$. In our experiments, we compare the standard auction and the auction with integer masses for $k=1,10,50,100$.

We generated normal instances with 1,000 to 10,000 points, in increments of 1,000, with 10 instances per size. Figure 9 shows the average running times. There is an overhead for mass 1 (a factor of roughly 4.5 in the figure). This ratio is not constant: the overhead becomes larger when the number of points grows. We also observe that it depends on the parameters of the distribution from which the points were drawn. For average mass 10, the auction with masses is comparable to the standard auction. For higher masses, 50 and 100, the advantage of the former is evident.

There is no clear dependence between the running time and the average mass. We took 4 instances with 10000 points each and tried larger average masses (with the same $[\lceil k / 2\rceil,\lfloor 3 k / 2\rfloor]$ distribution). Figure 10 illustrates the result. We can see that the running time does not increase much when the average mass increases, and may even decrease. That seems to depend very much on the particular instance and the distribution of masses inside it.

The memory consumption of auction with integer masses usually scales linearly with the number of points (for fixed average mass). In principle, the memory size can grow proportional to the total mass of the point sets when all slices shrink to size one, but such intensive slicing did not appear in our examples.

\section{Conclusion}

We have demonstrated that geometry helps to compute bottleneck and Wasserstein distances of bipartite point sets in two dimensions. Our approach leads to a faster computation of distances between persistence diagrams. Therefore, we expect our software to have an immediate impact on the computational pipeline of topological data analysis.

For bottleneck matchings, an interesting question would be how our k-d tree implementation compares in practice with the (theoretically) more time efficient, but more space demanding alternative of range trees, and with other point location data structures.

For Wasserstein matchings, we plan to further improve our implementation of the auction algorithm, including a parallel version for large instances. Simple heuristics can also improve special cases. For example, if $X$ and $Y$ are persistence diagrams and $S \subset X \cap Y$ is the set of common offdiagonal points, it holds for $q=1$ that $W_{q}(X, Y)=W_{q}(X \backslash S, Y \backslash S)$, as one shows very easily. This property allows to remove common points in the diagram before applying the auction algorithm. 

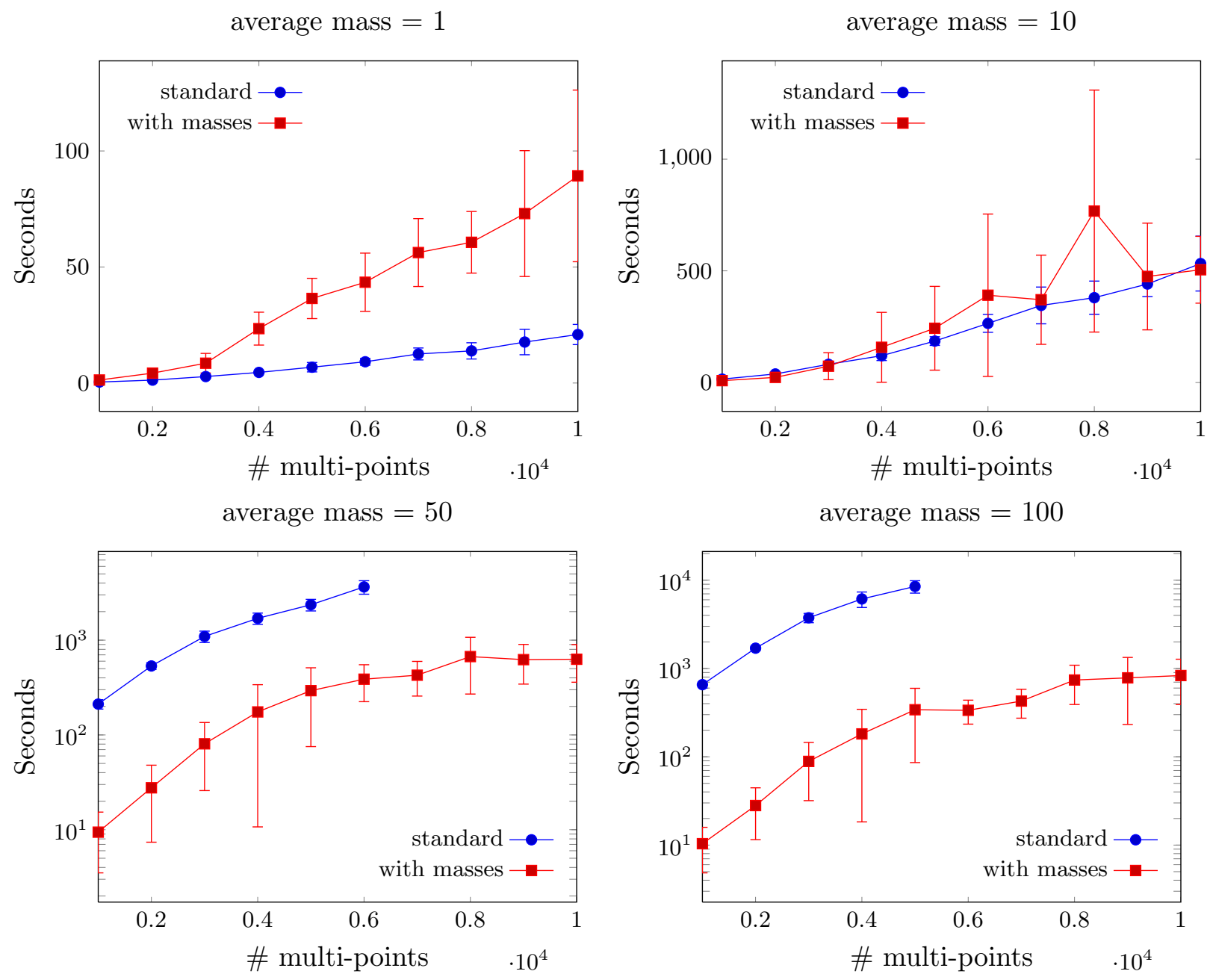

Figure 9: Comparison of standard auction and auction with integer masses on normal data for mass 1 (upper-left) and average masses 10 (upper-right), 50 (lower-left) and 100 (lower-right).

We also wonder how the auction approach compares with the various alternatives proposed in [9], and for which of these approaches can geometry help compute the Wasserstein distance efficiently, either exactly or approximately.

A natural approximation scheme for computing the Wasserstein distance for very large instances consists of placing a finite grid over $\mathbb{R}^{2}$ and "snapping" points to their closest grid vertex. The result is an instance with a potentially high multiplicity in each grid vertex. The problem with this approach is the approximation error introduced by the discretization step. A crude error bound is the total number of points in both diagrams multiplied by the diameter of the grid cells. An interesting question is to evaluate more refined discretization schemes with respect to their practical performance.

\section{Acknowledgements}

We thank Sergio Cabello for pointing out that the worst-case complexity of k-d trees and range trees remains valid under deletions of points, and for further valuable remarks on an earlier draft 


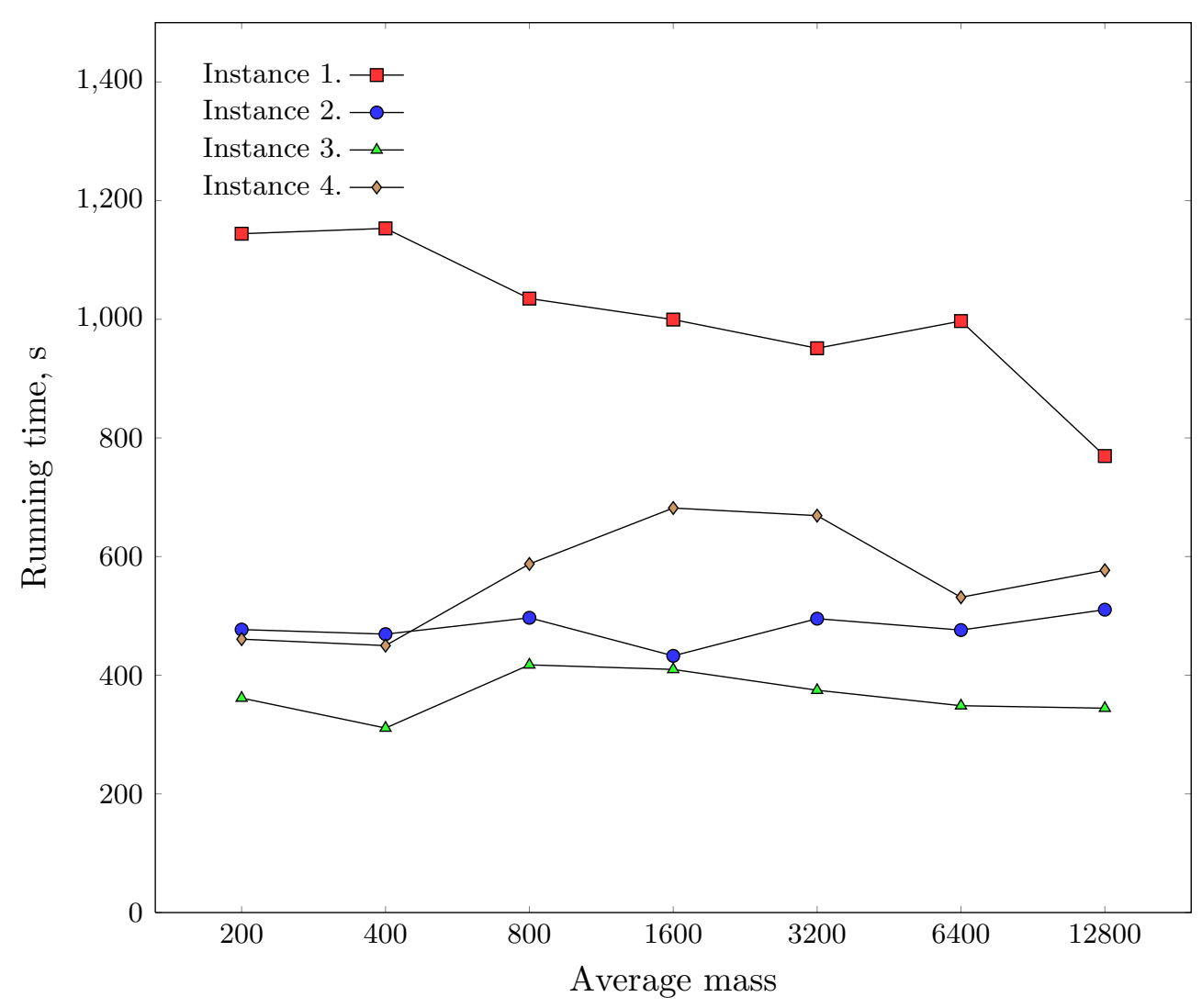

Figure 10: Dependence of the running time from the average mass for four particular instances of size 10000. Note the exponential scale on the $x$-axis.

of the paper.

Michael Kerber and Arnur Nigmetov acknowledge support by the Max Planck Center for Visual Computing and Communication. Dmitriy Morozov is supported by Advanced Scientific Computing Research, Office of Science, U.S. Department of Energy, under Contract DE-AC02-05CH11231.

\section{References}

[1] Aaron Adcock, Daniel Rubin, and Gunnar Carlsson. Classification of hepatic lesions using the matching metric. Computer Vision and Image Understanding, 121:36-42, 2014.

[2] Pankaj K. Agarwal and R. Sharathkumar. Approximation algorithms for bipartite matching with metric and geometric costs. In Symposium on Theory of Computing, STOC 2014, New York, NY, USA, May 31 - June 03, 2014, pages 555-564, 2014.

[3] Alexander Andrievsky and Andrei Sobolevskii. WANN: An implementation of weighted nearest neighbor search. Manual, available at http://www.mccme.ru/ ansobol/otarie/software. html, 2008.

[4] Jon L. Bentley. Multidimensional binary search trees used for associative searching. Communications of the ACM, 18:509-517, 1975. 
[5] Dimitri Bertsekas. A distributed algorithm for the assignment problem. Technical report, Laboratory for Information and Decision Sciences, MIT, 1979.

[6] Dimitri Bertsekas. The auction algorithm: A distributed relaxation method for the assignment problem. Annals of Operations Research, 14(1):105-123, 1988.

[7] Dimitri Bertsekas and David Castañon. The auction algorithm for the transportation problem. Annals of Operations Research, 20(1):67-96, 1989.

[8] Dimitri Bertsekas and David Castañon. Parallel synchronous and asynchronous implementations of the auction algorithm. Parallel Computing, 17(6):707-732, 1991.

[9] Rainer E. Burkard, Mauro Dell'Amico, and Silvano Martello. Assignment Problems, Revised Reprint:: Other titles in applied mathematics. Society for Industrial and Applied Mathematics (SIAM, 3600 Market Street, Floor 6, Philadelphia, PA 19104), 2009.

[10] L. Paul Chew and Klara Kedem. Improvements on geometric pattern matching problems. In Algorithm Theory - SWAT '92, Third Scandinavian Workshop on Algorithm Theory, Helsinki, Finland, July 8-10, 1992, Proceedings, pages 318-325, 1992.

[11] David Cohen-Steiner, Herbert Edelsbrunner, and John Harer. Stability of persistence diagrams. Discrete $\& 5$ Computational Geometry, 37(1):103-120, 2007.

[12] David Cohen-Steiner, Herbert Edelsbrunner, John Harer, and Yuriy Mileyko. Lipschitz functions have $L_{p}$-stable persistence. Foundations of Computational Mathematics, 10(2):127-139, 2010 .

[13] Mark de Berg, Marc van Kreveld, Mark Overmars, and Otfried Schwarzkopf. Computational Geometry: Algorithms and Applications. Springer, 2nd edition, 2000.

[14] Herbert Edelsbrunner and John Harer. Computational Topology. An Introduction. American Mathematical Society, 2010.

[15] Herbert Edelsbrunner, David Letscher, and Afra Zomorodian. Topological persistence and simplification. In 41st Annual Symposium on Foundations of Computer Science, FOCS 2000, 12-14 November 2000, Redondo Beach, California, USA, pages 454-463, 2000.

[16] Jack Edmonds. Paths, trees, and flowers. Canadian Journal of Mathematics, 17:449-467, 1965.

[17] Alon Efrat, Alon Itai, and Matthew J. Katz. Geometry helps in bottleneck matching and related problems. Algorithmica, 31(1):1-28, 2001.

[18] Jennifer Gamble and Giseon Heo. Exploring uses of persistent homology for statistical analysis of landmark-based shape data. Journal of Multivariate Analysis, 101(9):2184 - 2199, 2010.

[19] Chen Gu, Leonidas J. Guibas, and Michael Kerber. Topology-driven trajectory synthesis with an example on retinal cell motions. In International Workshop on Algorithms in Bioinformatics (WABI), pages 326-339, 2014.

[20] John E. Hopcroft and Richard M. Karp. An $n^{5 / 2}$ algorithm for maximum matchings in bipartite graphs. SIAM Journal on Computing, 2(4):225-231, 1973. 
[21] Michael Kerber, Dmitriy Morozov, and Arnur Nigmetov. Geometry helps to compare persistence diagrams. In Proceedings of the Eighteenth Workshop on Algorithm Engineering and Experiments, ALENEX 2016, Arlington, Virginia, USA, January 10, 2016, pages 103-112, 2016.

[22] Dmitriy Morozov. Dionysus library for computing persistent homology. mrzv.org/software/ dionysus, 2010.

[23] David M. Mount and Sunil Arya. ANN: A library for approximate nearest neighbor searching. http://www.cs.umd.edu/ mount/ANN, 2010.

[24] James Munkres. Algorithms for the assignment and transportation problems. Journal of the Society of Industrial and Applied Mathematics, 5(1):32-38, March 1957.

[25] Pravin M. Vaidya. Geometry helps in matching. SIAM J. Comput., 18(6):1201-1225, 1989.

[26] Anatoly Moiseevich Vershik. Long history of the Monge-Kantorovich transportation problem. The Mathematical Intelligencer, 35(4):1-9, 2013. 